\title{
Western strategy for EGJ carcinoma
}

\author{
Simone Giacopuzzi ${ }^{1}$ - Maria Bencivenga ${ }^{1} \cdot$ Jacopo Weindelmayer $^{1}$. \\ Giuseppe Verlato $^{2} \cdot$ Giovanni de Manzoni $^{1}$
}

Received: 10 October 2016/Accepted: 15 December 2016/Published online: 30 December 2016

(C) The International Gastric Cancer Association and The Japanese Gastric Cancer Association 2016

\begin{abstract}
In this paper, the epidemiological and clinicobiological behavior of esophagogastric junction (EGJ) adenocarcinoma in the West is compared and contrasted to that in the East, and an overview is provided of current therapeutic strategies employed for this type of tumor in Western countries. It is well known that multimodal treatment is the therapeutic standard in locally advanced EGJ adenocarcinoma, but whether neoadjuvant/perioperative chemotherapy (CT) or neoadjuvant chemoradiotherapy (CRT) is the optimal approach is still debated. Neoadjuvant CRT improves local control in locally advanced Siewert type I and II tumors, so it should be considered the treatment of choice. In the subset of these patients with microscopic systemic disease at diagnosis, more intensive exclusive chemotherapy protocols could be of benefit. Therefore, there is an urgent need to identify these patients before planning the treatment. For Siewert type III tumors, perioperative chemotherapy is the standard. While there is general agreement on the optimal surgical approach for Siewert types I and III (a two-field Ivor Lewis operation and a total gastrectomy with distal esophagectomy, respectively), no standard surgical treatment has been defined for Siewert type II tumors. When data from Western series on proximal and circumferential resection margins and on nodal spread in Siewert type II tumors are
\end{abstract}

Giovanni de Manzoni

giovanni.demanzoni@univr.it

1 General and Upper G.I. Surgery Division, Department of Surgery, University of Verona, Piazzale Stefani 1, 37126 Verona, Italy

2 Unit of Epidemiology and Medical Statistics, Department of Public Health and Community Medicine, University of Verona, Verona, Italy taken into account, the optimal surgical approach appears to be Ivor Lewis esophagectomy. Whether the extent of esophageal invasion can correctly predict nodal involvement in middle-upper mediastinal stations as a means to restrict indications for transthoracic esophagectomy requires further investigation in the West.

Keywords Gastroesophageal junction adenocarcinoma . Chemotherapy · Chemoradiotherapy · Ivor Lewis esophagectomy $\cdot$ Total gastrectomy

\section{Introduction}

The incidence of adenocarcinoma of the esophagogastric junction (EGJ) has increased rapidly over the last few decades, especially in Western countries. Indeed, in Europe and the US, EGJ tumors represent about one-third of all esophagogastric adenocarcinomas [1, 2], whereas this proportion does not exceed $10 \%$ in Far Eastern countries $[3,4]$. The different epidemiological trends seen for EGJ adenocarcinomas in different regions around the world reflect the geographical distributions of risk factors associated with EGJ, which are mainly related to the environment and lifestyle habits.

As it is a "zone disease" rather than an "organ disease," EGJ adenocarcinoma is etiologically heterogeneous. Indeed, an adenocarcinoma may develop in the transition zone between the esophagus and the stomach due to either neoplastic transformation of a metaplastic Barrett's mucosa caused by gastroesophageal reflux, malignant degeneration of proximal gastric mucosa (frequently related to $H$. pylori infection and gastric atrophia), or a neoplastic process primarily involving the transitional epithelium. These biological subtypes behave differently. Specifically, EGJ 
adenocarcinoma on Barrett's mucosa can be considered an esophageal cancer, while EGJ adenocarcinoma arising from the proximal gastric glands behaves like non-cardia gastric cancer, which has important therapeutic implications [5]. However, these tumors are morphologically indistinguishable, so the best way to differentiate them would be on a molecular basis, but there are unfortunately no validated molecular biomarkers that could be used to achieve this differentiation.

At present, EGJ adenocarcinomas are distinguished topographically using the well-known Siewert classification [6], which identifies three subtypes based upon the location of the center of the tumor with respect to the gastroesophageal junction. A type I tumor has its center 1-5 cm above the junction, a type II tumor has its center between $1 \mathrm{~cm}$ proximally and $1 \mathrm{~cm}$ distally from the EGJ, while a type III tumor has its center $1-5 \mathrm{~cm}$ distal from the junction. This topographic classification undoubtedly reflects the distinct etiologies and biological subtypes of Siewert type I and type III tumors. Indeed, it has been extensively reported that Siewert type I tumors arise from Barrett's intestinal metaplasia [5, 7], while Siewert type III cancers are associated with $H$. pylori infection and gastric atrophia [5]. But, for type II tumors, it is impossible to identify a univocal etiopathogenetic mechanism. While all tumors that are centered on the EGJ are topographically classified as Siewert type II, they are actually a mixture of esophageal adenocarcinomas arising on short or ultrashort Barrett's esophagus, gastric cancers originating from the most proximal gastric mucosa, and "true" cardia cancers [5]. Therefore, among EGJ adenocarcinomas, Siewert type II tumors are the most problematic when attempting to accurately identify the tumor's biological subtype and thus select the most appropriate treatment.

When Eastern and Western EGJ adenocarcinoma series are compared according to the Siewert classification, very interesting dissimilarities between them can be observed. First, the proportions of the three subtypes are very different in Eastern and Western series: the proportions are almost equal in European series, whereas most EGJ tumors in Korea and Japan are Siewert types II and III (the proportion of Siewert type I is $<4 \%$ ) [8]. These differences are very likely due to the higher prevalence of reflux-related conditions in Western countries.

There is also a detectable difference in the biological characteristics of tumors that Eastern and Western surgeons classify as Siewert type II. Indeed, in Eastern series, in terms of the prognosis, Siewert II tumors have similar oncological outcomes to Siewert III tumors, and no differences between EGJ and non-cardia gastric cancer are seen [9]. Conversely, in Western countries, the three Siewert subtypes have different prognoses: good in Siewert type I tumors, intermediate for Siewert II tumors, and poor (comparable to that of non-cardia gastric cancer) in Siewert type III tumors [10].

Therefore, it is likely that in Western countries, where gastroesophageal reflux is more prevalent than in most (but not all) Eastern countries, Siewert type II tumors arise from short or ultrashort Barrett's mucosa, so most of them behave as esophageal cancers. Conversely, in countries with a high incidence of non-cardia gastric cancer, namely Korea and Japan, most junctional type II cancers are proximal gastric tumors invading the distal esophagus.

Moreover, in Western countries, except for monitoring for Barrett's esophagus, there are no widespread screening programmes for the early detection of upper GI tumors, meaning that most EJG adenocarcinomas are diagnosed at an advanced stage.

Taken together, these considerations indicate that Eastern and Western surgeons face different realities for EGJ adenocarcinomas. In the West, one in three patients with esophagogastric adenocarcinoma is diagnosed with an advanced EJG tumor, most of which behave as an esophageal adenocarcinoma. In the East, only one in ten patients with esophagogastric adenocarcinoma have a tumor located at the EGJ; of those that are, most are early cancers that are biologically similar to non-cardia gastric cancer. Thus, the East and West perceive EGJ adenocarcinomas from different points of view.

In this article, we provide an overview of the current strategy for EGJ carcinomas in the West, focusing on the treatment of locally advanced forms, which represent the majority of the cases.

\section{Multimodal treatment of EGJ adenocarcinoma in the West}

In cases of EGJ adenocarcinoma, the treatment strategy is tailored on the basis of the primary staging. A precise endoscopic description of esophageal and gastric invasion of the tumor, defining the Siewert type, is of paramount importance for therapeutic decisions. Note that endoscopy has significant limitations when attempting to accurately determine the extent of esophageal invasion and the Siewert type of the tumor. In particular, some difficulties in distinguishing between type II and III tumors have been observed: endoscopy has a very low specificity (44\%) when classifying Siewert type II tumors [11]. Moreover, EUS and CT scan are necessary to define lymph node involvement and to rule out the presence of distant metastasis.

Although surgery is the mainstay of treatment, survival after radical resection in cases of locally advanced EGJ adenocarcinoma is reported to be poor [12], so the multimodal approach is currently considered the standard. 
Neoadjuvant therapy: chemotherapy versus chemoradiotherapy

There does not appear to be any notable survival benefit of adjuvant chemotherapy [13] in esophageal cancer. The addition of adjuvant chemoradiotherapy (CRT) to surgery in cases of EGJ and gastric adenocarcinoma was investigated in the INT-0116 phase III trial [14]; adjuvant CRT was reported to give a survival benefit over surgery alone, but the advantage of postoperative treatment was questionable if adequate surgery was performed [15]. A number of investigations assessing the role of neoadjuvant treatments have therefore been performed in the West over the last few decades.

Both pre/perioperative chemotherapy and preoperative CRT show advantages compared to surgery alone in cases of EGJ adenocarcinoma [16].

As regards the role of perioperative chemotherapy, the two largest phase III trials-MAGIC and ACCORD [17, 18]—demonstrated a significant improvement in 5-year overall survival for the combined approach compared to surgery alone in locally advanced gastric and EGJ adenocarcinomas (5-year OS 36 vs $23 \%$ in MAGIC and 38 vs $24 \%$ in ACCORD). The R0 resection rate was similar in the perioperative chemotherapy and surgery-alone groups (69.3 and $66.4 \%$, respectively). Note that only one in four patients in the MAGIC trial had a tumor located at the EGJ, while $75 \%$ of the patients enrolled in the French ACCORD trial had EGJ tumors. That said, in both of those trials, subgroup analyses showed that the largest beneficial effect of perioperative chemotherapy occurred for EGJ tumors, bearing in mind that all Siewert types were included in both studies. The main limitation of these two trials is that global survival was only rarely reported in the surgeryalone arms, very likely due to inadequate quality of surgical procedure together with inaccurate preoperative staging.

A survival advantage of pre/perioperative chemotherapy over surgery alone in gastroesophageal adenocarcinoma and squamous cell carcinoma (SCC) of the esophagus was also confirmed in a meta-analysis by Sjoquist et al. [16].

As for preoperative CRT, the most relevant study is the CROSS trial [19], in which patients with esophageal and EGJ adenocarcinoma of Siewert type I or II $(75 \%)$ and SCC of the esophagus (25\%) were randomly assigned to either weekly carboplatin and paclitaxel with concurrent radiotherapy (41.4 Gy given in 23 fractions of $1.8 \mathrm{~Gy}$ on 5 days per week) followed by surgery or surgery alone. An impressive R0 resection rate of $92 \%$ after neoadjuvant CRT (compared to $69 \%$ after surgery) was reported; $29 \%$ of patients who underwent resection after neoadjuvant CRT showed a pathologic complete response (pCR), this rate was $23 \%$ when only adenocarcinoma patients were considered. Follow-up data showed that CRT significantly reduced both locoregional and distant recurrences compared to surgery alone [20]. While both univariate and multivariate analyses indicated a significant overall survival benefit of neoadjuvant CRT in SCC, the median overall survival improved with multimodal treatment in esophageal and EGJ adenocarcinoma: 43.2 months compared to 27.1 months after surgery alone. This difference was significant in univariate analysis $\mathrm{HR}(0.73 ; 95 \%$ CI $0.55-0.98, p=0.037$ ) but nonsignificant in multivariate HR $(0.75$; 95\% CI 0.56-1.01, $p=0.059)$ [20].

In phase I and II trials implemented by our group [21-23], Siewert type I and II EGJ adenocarcinomas were treated with neoadjuvant CRT; they received 3 weeks of induction chemotherapy alone (5-fluorouracil administered by protracted intravenous infusion plus weekly administration of iv cisplatin and docetaxel) followed by $50.4 \mathrm{~Gy}$ of concurrent radiotherapy. Results showed an R0 resection rate of $88 \%$ and a pathologic complete response rate of $40 \%$. A 5 -year overall survival of $43 \%$ was reported for the entire series of patients treated with our protocol, including those with esophageal SCC and EGJ adenocarcinomas. The 5-year disease-related survival was $49 \%$, and the long-term survival was better in adenocarcinoma cases than in SCC cases [23].

The higher rate of complete response and the better overall survival reported by our group when compared with data from the CROSS trial suggest that the application of more intensive chemotherapy protocols and an increased radiotherapy dose could improve the results of preoperative CRT.

There is still some controversy over whether neoadjuvant/perioperative chemotherapy (CT) or neoadjuvant chemoradiotherapy (CRT) is the optimal neoadjuvant strategy for EGJ adenocarcinoma. Chemoradiotherapy yields higher rates of $\mathrm{R} 0$ resection and $\mathrm{pCR}$, while chemotherapy hypothetically exerts a greater systemic effect than CRT due to the low radiosensitizing dose of systemic chemotherapy that such treatments permit [24]. This systemic effect would act on circulating micrometastases detected in a nonnegligible proportion of the patients undergoing surgery for esophageal cancer [25].

Few studies have directly compared neoadjuvant chemotherapy to chemoradiotherapy [26-28]. Similar to previous reports, the most recent comparative study-the NeoRes randomized trial—showed a higher R0 resection rate after CRT compared to the chemotherapy group ( $87 \mathrm{vs}$ $74 \%, p=0.04)$ and a higher histologic complete response rate, which was $28 \%$ after CRT vs $9 \%$ after chemotherapy $(p=0.002)$, but it failed to demonstrate a difference in overall survival between the two treatment arms [28]. One hypothesis to explain the lack of a clear survival advantage when comparing the two approaches is that, despite 
providing better local control of the disease, neoadjuvant CRT is not the best approach in all patients; some of them, namely those who have microscopic systemic disease at diagnosis, may be better treated with exclusive chemotherapy.

Interestingly, in series of EGJ adenocarcinoma cases treated with preoperative CRT, it was found that pCR patient relapses are often systemic [23, 29].

Thus, based on the available data, neoadjuvant CRT definitely improves local control of locally advanced EGJ adenocarcinomas of Siewert type I and II, and should therefore be considered the treatment of choice in this clinical setting. More intensive CRT treatments are thought to further improve CRT efficacy. However, in patients with microscopic systemic disease at diagnosis, the local effect of CRT is not enough, and more intensive exclusive chemotherapy schedules may be beneficial. There is, therefore, an urgent need to identify this subgroup of patients before choosing the treatment plan.

As regards Siewert type III tumors (which are only rarely considered in trials of preoperative CRT), the strongest evidence come from the MAGIC and ACCORD trials $[17,18]$. Thus, the optimal multimodal approach in Siewert type III tumors is currently pre/perioperative chemotherapy.

Another important issue is the evidence of primary chemo- and radioresistance of EGJ adenocarcinoma, including all Siewert types [24]. To avoid the toxicity resulting from ineffective chemotherapy or chemoradiation treatment, there is an urgent need to identify biomarkers that are predictive of the response of a tumor to chemotherapy and radiotherapy. Recently, our group identified a novel promising biomarker of chemoradioresistance in EGJ Siewert type I and II adenocarcinomas [30]. These biomarkers should soon be validated in the clinical setting, thus allowing the selection of responsive patients before preoperative therapy.

\section{EGJ signet ring cell carcinomas}

As regards resistance to preoperative treatments, "signet ring" cell (SRC) carcinomas require special consideration. These tumors are of great interest due to both their increasing incidence in recent decades and their biological aggressiveness [31, 32]. Nafteux et al. [32] recently reported the outcomes of their EGJ adenocarcinoma series treated with primary surgery according to histological features: the group of patients with SRC-containing histology showed poor prognosis compared to the group of patients with adenocarcinoma but without SRCs: the cancer-specific 5-year survival was $22.4 \%$ vs $59.3 \%$, respectively. This prognostic difference was even more evident when only the group of patients with true SRC carcinoma
(SRC component $>50 \%$ according to WHO classification) was considered. Based on this evidence, surgery alone is ineffective, so a more aggressive strategy is needed. Neoadjuvant chemotherapy resulted in a low rate of response in SRC EGJ tumors compared to non-SRC EGJ tumors (approximately 21 and 17\%, respectively): pCR was observed in only $3.4 \%$ of the SRC EGJ tumor cases [33]. However, if a response to therapy is observed, it is associated with a better prognosis [33]. Neoadjuvant CRT yields a higher rate of pathologic complete response than chemotherapy [34]. Moreover, CRT was the only independent favorable prognostic factor in a setting of locally advanced SRC EGJ tumors [35]. That said, the available data are still insufficient; dedicated randomized trials or subgroup analyses stratified according to tumor histology are needed.

\section{Clinical T2NO tumors}

A further issue to consider is the correct management of clinically staged T2N0 (cT2N0) tumors, which constitute approximately $5-15 \%$ of the cases in Western series $[36,37]$. Considering the limited extent of the disease in these cases, preoperative therapies-burdened by negligible morbidity - could represent overtreatment. Despite these considerations, there has been a tendency in more recent years to treat a greater number of cT2N0 patients with multimodal neoadjuvant approaches [35], given that it reportedly yields better biological control of the disease $(6-18 \%$ of cT2N0 patients treated with multimodal therapy were pathologic complete responders) and a higher rate of radical resection [36-38]. However, any observed advantage in terms of overall and disease-free survival comes from comparing multimodality therapy with surgery alone in this clinical setting [36-38]. It should be noted that these results cannot be considered to be conclusive, especially because a nonnegligible percentage of clinical N0 cases are actually pathological $\mathrm{N}+(40-50 \%$ of such cases are upstaged in upfront surgery) [36-38], and those cases could therefore benefit from neoadjuvant treatments [38]. For this reason, parameters such as tumor grade and lymphovascular invasion should be taken into account along with clinical staging when attempting to predict nodal invasion and to identify potentially understaged patients before choosing the treatment plan [38].

\section{EGJ adenocarcinoma surgery in the West}

Unlike esophageal SCC, the surgical strategy for EGJ adenocarcinoma is either gastrectomy and distal esophagectomy with an exclusive abdominal approach or transthoracic Ivor Lewis esophagectomy. 
Curative (R0) resection is a mainstay of surgery, as it guarantees the best survival outcome. Indeed, survival after noncurative surgery is reported to be very poor [39].

The optimal extent of surgical resection is selected based upon the need for clear longitudinal and circumferential resection margins and also upon the adequacy of lymphadenectomy, considering the nodal spread of the tumor. Note that surgical strategy is decided on the basis of the primary staging, even in tumors treated with a neoadjuvant therapy.

\section{Resection margins}

The macroscopic negativity of longitudinal margins is not a sufficient criterion for radicality in cases of EGJ adenocarcinoma, given its tendency to spread intramurally with the mucosal layer uninvolved, either as a direct extension of the primary tumor or as a distant metastasis [40, 41]. Many studies have been conducted to define the optimal extent of longitudinal margins, using measures from in vivo or fixed specimens. It should be noted that the esophagus shrinks immediately after the resection, resulting in a reduction of $10-45 \%$ in the in vivo length $[42,43]$. As regards the proximal margin, in 2003 Mariette published a study on Siewert type I and II tumors and proposed an $8-\mathrm{cm}$ in situ margin, considering a shrinkage of $50 \%$, thus doubling all the fresh contracted gross specimen measures [44]. Ito et al. defined a proximal margin length of $6 \mathrm{~cm}$ as safe, whilst in 2007 Barbour observed improved survival with an in vivo proximal margin of about $5 \mathrm{~cm}$ (3.8 $\mathrm{cm}$ in the specimen) $[45,46]$.

Determining the distal margin to use for EGJ adenocarcinoma can be problematic, especially for true cardia cancers with extensive invasion of both the esophageal and gastric sides. Indeed, an esophagectomy is required in this case, but gastric tubulization could hinder the attainment of a safe distal margin. In the aforementioned study by Ito, a negative distal margin was found in all patients, with at least $4 \mathrm{~cm}$ of macroscopically free stomach below the tumor, while a $5-\mathrm{cm}$ in vivo distal margin was advocated in a study from the UK $[45,47]$.

To summarize, proximal $5-6 \mathrm{~cm}$ and distal $4-5 \mathrm{~cm}$ in vivo margins are currently considered safe for EGJ adenocarcinoma.

\section{Circumferential resection margin}

In recent years, the concept of the circumferential resection margin (CRM) - widely studied for rectal cancer-has also been gaining interest in relation to esophageal tumors: both squamous cell cancers and esophageal and EGJ adenocarcinomas. Two classifications have been proposed for the CRM: the College of American Pathologists (CAP) considers the CRM to be positive only if the tumor involves the surgical margin, while The Royal College of Pathologists (RCP) define the CRM as positive if the tumor is found within $1 \mathrm{~mm}$ from the margin. The CRM only needs to be studied for pT3 tumors because a positive CRM for an earlier-stage tumor means that surgery was inadequate and the CRM is always positive in pT4 tumors. Data on the CRM in esophageal cancer were collected and evaluated in two recent meta-analyses [48, 49]. The results indicated an $\mathrm{R}+$ rate of $15-17 \%$ with the CAP criteria and 36-40\% when utilizing the RCP criteria, with significant reductions in survival in both the CAP and RCP R+ groups. Patients with a positive RCP CRM but a negative CAP CRM had a significantly poorer outcome than CRM negative patients according to both RCP and CAP CRM definition, and thus probably represent an intermediate-risk group of patients.

Neoadjuvant chemoradiation can theoretically reduce the impact of the CRM on the prognosis due to the local control of the disease obtained with such treatment. In the meta-analysis conducted by Chan and colleagues, a subgroup analysis was carried out on patients who received chemoradiation. The results showed a significant reduction in OS in the CRM+ group for both classifications, but when only patients with an involvement within $1 \mathrm{~mm}$ from the margin were considered, the difference was no longer significant [48]. Although only preliminary, these results confirm the influence of CRT on microscopically involved CRM. In these patients, CAP is probably more informative than RCP classification.

In terms of the impact of the surgical approach on the CRM in EGJ adenocarcinoma, a study from the Netherlands showed a significantly lower CRM+ rate, according to the CAP classification, after esophagectomy compared to gastrectomy [50].

\section{Lymph node metastasis and lymphadenectomy}

As regards the spread of EGJ adenocarcinoma to lymph nodes, the three Siewert types show significant differences, reflecting their different tumor biologies. It should be noted that the incidence of metastasis reported for each node station varies among series, depending on the type of surgical resection performed. The distribution of mediastinal and abdominal metastases for each Siewert type, as reported by Western authors, is described below and summarized in Table 1.

In Siewert type I tumors, when standard mediastinal lymphadenectomy is performed, about half of the nodepositive patients show positive nodes in mediastinal stations [51]. Specifically, upper and mid mediastinal node metastases (right paratracheal, subcarinal, aortopulmonary window) are reported in up to $25 \%$ of patients 
Table 1 Nodal spread of EGJ adenocarcinoma in Western series

\begin{tabular}{|c|c|c|c|}
\hline Author/country & $\begin{array}{l}\text { No. of patients/Siewert } \\
\text { type }\end{array}$ & $\begin{array}{l}\text { Surgical approach for each Siewert } \\
\text { type }\end{array}$ & Rate of mediastinal node metastasis \\
\hline $\begin{array}{l}\text { Hulscher, } 2001 \text { (Germany) } \\
\text { [53] }\end{array}$ & $\begin{array}{l}74 \text { patients } \\
60 \mathrm{SI} \\
14 \mathrm{SII}\end{array}$ & TTE two-field lymphadenectomy & $\begin{array}{l}\text { Upper mediastinal: } 8 \% \\
\text { Mid mediastinal: } 19 \% \\
\text { Lower med/paracardial: } 47 \%\end{array}$ \\
\hline $\begin{array}{l}\text { de Manzoni, } 2007 \text { (Italy) } \\
\text { [51] }\end{array}$ & $\begin{array}{l}143 \text { patients } \\
20 \text { SI } \\
62 \text { SII } \\
61 \text { SIII }\end{array}$ & $\begin{array}{l}\text { SI: TTE } \\
\text { SII: TTE or TG } \\
\text { SIII: } 97 \% \text { TG, } 3 \% \text { TTE }\end{array}$ & $\begin{array}{l}\text { SI: upper-mid mediastinal } 5 \% \text {, lower medistinal } \\
25 \% \\
\text { SII: upper-mid mediastinal } 4 \% \text {, lower medistinal } \\
13 \% \\
\text { SIII: lower mediastinal } 5 \%\end{array}$ \\
\hline $\begin{array}{l}\text { Parry, } 2015 \text { (Netherlands) } \\
\text { [50] }\end{array}$ & $\begin{array}{l}266 \text { patients } \\
67 \mathrm{SI} \\
176 \mathrm{SII} \\
16 \mathrm{SIII}\end{array}$ & $\begin{array}{l}\text { SI: TTE } \\
\text { SII: } 88 \% \text { TTE, } 12 \% \text { TG } \\
\text { SIII: } 88 \% \text { TG, } 12 \% \text { TTE }\end{array}$ & $\begin{array}{l}\text { SI: upper-mid mediastinal } 25 \% \text {, lower } \\
\text { mediastinal } 33 \% \\
\text { SII: upper-mid mediastinal } 11 \% \text {, lower } \\
\text { mediastinal } 23 \%\end{array}$ \\
\hline $\begin{array}{l}\text { Lagarde, } 2005 \text { (Netherlands) } \\
\text { [58] }\end{array}$ & $\begin{array}{l}50 \text { patients } \\
\text { all SII }\end{array}$ & TTE & Upper-mid mediastinal: $22 \%$ \\
\hline
\end{tabular}

SI Siewert type 1, SII Siewert type II, SIII Siewert type III, TTE transthoracic esophagectomy, TG total gastrectomy

$[50,52,53]$. When the few studies in which cervical nodes are also removed $[54,55]$ are considered, a nonnegligible incidence of metastases is detectable, but, due to the scarcity of the data available and the higher complication rate of three-field dissection, this is not currently thought to be relevant in clinical practice. In terms of abdominal spread, first-tier stations are the most likely to be involved, although nodes around the celiac trunk were also found to be positive in up to $27 \%$ of locally avanced cases [56].

Mediastinal nodes are involved in about $30 \%$ of Siewert type II cases. Although most of the positive nodes occur at lower stations, the rate of metastasis detected in the upper-mid mediastinum after transthoracic esophagectomy ranges between 8 and 22\% [53, 57, 58]. On the abdominal side, the perigastric nodes are involved in all node-positive Siewert II tumors, while metastatic nodes at second-level stations are detected in up to $30 \%$ of cases [51]. As regards paraaortic nodal invasion, Eastern authors report a rate of metastasis of $16-17 \%$ in Siewert type II tumor cases [59]. These findings could be partly due to the different biology of Siewert type II tumors in the East, where most junctional tumors show similar biological behavior to non-cardia gastric cancers. However, Western authors also reported a nonnegligible $10 \%$ of paraaortic nodal metastases in type II EGJ adenocarcinoma cases [60], supporting previously reported studies on lymphatic flow from the cardias that showed a direct metastatic route from the left paracardial region to paraaortic nodes, specifically around the left renal vein (16a2 station) [61, 62], and thus the need to extend the lymphadenectomy beyond the standard D2 dissection to paraaortic nodes in this tumor type.

Note that, in our series [51], as well as in Eastern reports $[63,64]$, the rate of nodal metastasis along the greater curvature (stations $4 \mathrm{sa}-4 \mathrm{sb}-4 \mathrm{~d}$ ) is very low in Siewert type II tumors, suggesting that a gastric tube can be used for reconstruction after transthoracic esophagectomy.

Positive mediastinal nodes are found in about $10 \%$ of all Siewert type III tumor cases, and lower stations are involved in almost all of those positive cases. Abdominal perigastric and second-tier nodes are the main sites of lymphatic metastases in Siewert type III tumor cases, and positive paraaortic nodes are found in up to $30 \%$ of cases after a D3 dissection [51].

There is general agreement among Western authors on the optimal surgical approach in Siewert type I and III tumors-esophagectomy with a two-field Ivor Lewis operation and a total gastrectomy with distal esophagectomy with lower mediastinal lymphadenectomy through an exclusive abdominal approach, respectively. However, no standard surgical approach has been defined for Siewert type II tumors. Indeed, some European authors still maintain that transhiatal total gastrectomy is the optimal surgical approach [52], while many other [23, 46] Western surgeons consider a two-field Ivor Lewis operation to be mandatory.

Based on the above-reported data on proximal and cirumferential resection margins and nodal spread in Siewert type II tumors from Western series, the optimal surgical approach to ensure a radical resection should be the two-field Ivor Lewis esophagectomy. However, due to 
the different morbidities and mortalities of an exclusive abdominal operation and a two-field approach, many studies are focusing on the best way to select patients that can be safely treated with a less invasive approach.

Interestingly, as the rate of mediastinal node metastasis increases with increasing esophageal invasion [65-67], some authors suggest that the risk of upper-mid mediastinal node metastasis should be evaluated based on the extent of esophageal invasion, in order to limit transthoracic esophagectomies in EGJ tumor cases, which have a consistent risk of nodes that are not dissectable through an exclusive abdominal approach. Specifically, Eastern authors [68] reported a significantly higher risk of metastases in right paratracheal, subcarinal, and aortopulmonary window nodes if the esophageal invasion is more than $3 \mathrm{~cm}$.

The differences between the East and West in EGJ tumor biology and treatment approaches must be taken into account before applying the results from Eastern trials to Western populations. Based on our personal experience (G. de Manzoni, unpublished data reported at the 2016 ESDE meeting, Munich, Germany, 1-3 December 2016) of our series of Siewert type I and II tumors, the length of esophageal involvement does not influence the prediction of the positivity of superior-middle mediastinal nodes, as some patients with limited $(<2 \mathrm{~cm})$ esophageal invasion were found to have positive nodes at these stations.

In order to avoid suboptimal surgery, in Siewert I and II cases with limited esophageal invasion, the pattern of nodal spread toward the mediastinum needs to be further evaluated in relation to the biological subtype and histology of the tumor in Western populations.

\section{Conclusions}

In conclusion, multimodal treatment is mandatory for EGJ adenocarcinoma. However, whether chemotherapy or CRT should be considered the best treatment choice is still debated.

Since neoadjuvant CRT was reported to significantly improve local control in locally advanced Siewert type I and II EGJ adenocarcinomas, it should be considered the treatment of choice for these tumors. Anyway, in a subset of these patients with microscopic systemic disease at diagnosis, more intensive exclusive CT protocols could be of benefit, so there is an urgent need to identify these patients before planning the treatment. For Siewert III tumors, perioperative chemotherapy is the standard.

As regards the surgical treatment, there is general agreement about the optimal surgical approach for Siewert types I and III-esophagectomy with a two-field Ivor Lewis operation and a total gastrectomy with distal esophagectomy through an abdominal approach, respectively; however, there is no standard surgical approach for Siewert type II tumors. When data from Western series on proximal and circumferential resection margins as well as on nodal spread in Siewert type II tumors are considered, the optimal surgical approach seems to be two-field Ivor Lewis esophagectomy. Whether the extent of esophageal invasion can correctly predict the nodal involvement in mid-upper mediastinal stations as a means to restrict indications for transthoracic esophagectomy requires further investigation in the West.

\section{Compliance with ethical standards}

Conflict of interest The authors declare that they have no conflict of interest.

Ethical standards This article does not contain any studies with human or animal subjects performed by any of the authors.

\section{References}

1. Carneiro F, Moutinho C, Pera G, et al. Pathology findings and validation of gastric and esophageal cancer cases in a European cohort (EPIC/EUR-GAST). Scand J Gastroenterol. 2007;42(5):618-27.

2. Wu HY, Rusiecki JA, Zhu KM, et al. Stomach carcinoma incidence patterns in the United States by histologic type and anatomic site. Cancer Epidemiol Biomark Prev. 2009;18(7): 1945-52.

3. Kusano C, Gotoda T, Khor CJ, et al. Changing trends in the proportion of adenocarcinoma of the esophagogastric junction in a large tertiary referral center in Japan. J Gastroenterol Hepatol. 2008;23(11):1662-5.

4. Lee JY, Kim HY, Kim KH, Jang HJ, Kim JB, Lee JH, et al. No changing trends in incidence of gastric cardia cancer in Korea. J Korean Med Sci. 2003;18:53-7.

5. McColl KE, Going JJ. Aetiology and classification of adenocarcinoma of the gastro-oesophageal junction/cardia. Gut. 2010;59(3):282-4.

6. Siewert JR, Stein HJ. Classification of adenocarcinoma of the oesophagogastric junction. Br J Surg. 1998;85:1457-9.

7. Leers JM, DeMeester SR, Chan N, Ayazi S, Oezcelik A, Abate E, et al. Clinical characteristics, biologic behavior, and survival after esophagectomy are similar for adenocarcinoma of the gastroesophageal junction and the distal esophagus. J Thorac Cardiovasc Surg. 2009;138(3):594-602.

8. Hasegawa S, Yoshikawa T. Adenocarcinoma of the esophagogastric junction: incidence, characteristics, and treatment strategies. Gastric Cancer. 2010;13(2):63-73.

9. Suh Y-S, Han D-S, Kong S-H, Lee H-J, Kim YT, Kim W-H, et al. Should adenocarcinoma of the esophagogastric junction be classified as esophageal cancer? A comparative analysis according to the seventh AJCC TNM classification. Ann Surg. 2012;255(5):908-15.

10. Reynolds JV, Ravi N, Muldoon C, Larkin JO, Rowley S, O'Byrne $\mathrm{K}$, et al. Differential pathologic variables and outcomes across the spectrum of adenocarcinoma of the esophagogastric junction. World J Surg. 2010;34(12):2821-9.

11. Pedrazzani C, Bernini M, Giacopuzzi S, Pugliese R, Catalano F, Festini M, et al. Evaluation of Siewert classification in gastro- 
esophageal junction adenocarcinoma: what is the role of endoscopic ultrasonography? J Surg Oncol. 2005;91(4):226-31.

12. Siewert JR, Feith M, Werner M, Stein HJ. Adenocarcinoma of the esophagogastric junction: results of surgical therapy based on anatomical/topographic classification in 1002 consecutive patients. Ann Surg. 2000;232(3):353-61.

13. Bhansali MS, Vaidya JS, Bhatt RG, Patil PK, Badwe RA, Desai PB. Chemotherapy for carcinoma of the esophagus: a comparison of evidence from meta-analyses of randomized trials and of historical control studies. Ann Oncol. 1996;7:355-9.

14. Macdonald JS, Smalley SR, Benedetti J, Hundahl SA, Estes NC, Stemmermann GN, Haller DG, Ajani JA, Gunderson LL, Jessup JM, Martenson JA. Chemoradiotherapy after surgery compared with surgery alone for adenocarcinoma of the stomach or gastroesophageal junction. N Engl J Med. 2001;345(10):725-30. doi:10.1056/NEJMoa010187.

15. Dikken JL, Jansen EP, Cats A, Bakker B, Hartgrink HH, Kranenbarg EM, Boot $\mathrm{H}$, Putter H, Peeters KC, van de Velde CJ, Verheij M. Impact of the extent of surgery and postoperative chemoradiotherapy on recurrence patterns in gastric cancer. J Clin Oncol. 2010;28(14):2430-6.

16. Sjoquist KM, Burmeister BH, Smithers BM, Zalcberg JR, Simes RJ, Barbour A, et al. Survival after neoadjuvant chemotherapy or chemoradiotherapy for resectable oesophageal carcinoma: an updated meta-analysis. Lancet Oncol. 2011;12(7):681-92.

17. Cunningham D, Allum WH, Stenning SP, et al. Perioperative chemotherapy versus surgery alone for resectable gastroesophageal cancer. N Engl J Med. 2006;355:11-20.

18. Ychou M, Boige V, Pignon JP, et al. Perioperative chemotherapy compared with surgery alone for resectable gastroesophageal adenocarcinoma: an FNCLCC and FFCD multicenter phase III trial. J Clin Oncol. 2011;29:1715-21.

19. van Hagen P, Hulshof MC, van Lanschot JJ, et al. Preoperative chemoradiotherapy for esophageal or junctional cancer. N Engl J Med. 2012;366:2074-84.

20. Shapiro J, van Lanschot JJB, Hulshof M, for the CROSS study group, et al. Neoadjuvant chemoradiotherapy plus surgery versus surgery alone for oesophageal or junctional cancer (CROSS): long-term results of a randomised controlled trial. Lancet Oncol. 2015;16:1090-8.

21. Pasini F, de Manzoni G, Pedrazzani C, Grandinetti A, Durante E, Gabbani M, et al. High pathological response rate in locally advanced esophageal cancer after neoadjuvant combined modality therapy: dose finding of a weekly chemotherapy schedule with protracted venous infusion of 5-fluorouracil and dose escalation of cisplatin, docetaxel. Ann Oncol. 2005;16(7):1133-9.

22. Pasini F, et al. Neoadjuvant therapy with weekly docetaxel and cisplatin, 5-fluorouracil continuous infusion, and concurrent radiotherapy in patients with locally advanced esophageal cancer produced a high percentage of long-lasting pathological complete response: a phase 2 study. Cancer. 2013;119(5):939-45.

23. Zanoni A, et al. Neoadjuvant concurrent chemoradiotherapy for locally advanced esophageal cancer in a single high-volume center. Ann Surg Oncol. 2013;20(6):1993-9.

24. Davies AR, Gossage JA, Zylstra J, et al. Tumor stage after neoadjuvant chemotherapy determines survival after surgery for adenocarcinoma of the esophagus and esophagogastric junction. J Clin Oncol. 2014;32:2983-90.

25. Bonavina L, Soligo D, Quirici N, Bossolasco P, Cesana B, Lembertenghi Deliliers G, Peracchia A. Bone marrow-disseminated tumor cells in patients with carcinoma of the esophagus or cardia. Surgery. 2001;129(1):15-22.

26. Stahl M, Walz MK, Stuschke M, et al. Phase III comparison of preoperative chemotherapy compared with chemoradiotherapy in patients with locally advanced adenocarcinoma of the esophagogastric junction. J Clin Oncol. 2009;27:851-6.

27. Burmeister BH, Thomas JM, Burmeister EA, et al. Is concurrent radiation therapy required in patients receiving preoperative chemotherapy for adenocarcinoma of the oesophagus? A randomised phase II trial. Eur J Cancer. 2011;47:354-60.

28. Klevebro F, Alexandersson von Döbeln G, Wang N, et al. A randomized clinical trial of neoadjuvant chemotherapy versus neoadjuvant chemoradiotherapy for cancer of the oesophagus or gastro-oesophageal junction. Ann Oncol. 2016;27:660-7.

29. van Hagen P, Wijnhoven BP, Nafteux P, Moons J, Haustermans $\mathrm{K}$, De Hertogh G, van Lanschot JJ, Lerut T. Recurrence pattern in patients with a pathologically complete response after neoadjuvant chemoradiotherapy and surgery for oesophageal cancer. $\mathrm{Br} \mathbf{J}$ Surg. 2013;100(2):267-73.

30. Piro G, Giacopuzzi S, Bencivenga M, Carbone C, Verlato G, Frizziero M, Zanotto M, Mina MM, Merz V, Santoro R, Zanoni A, De Manzoni G, Tortora G, Melisi D. TAK1-regulated expression of $\mathrm{BIRC} 3$ predicts resistance to preoperative chemoradiotherapy in oesophageal adenocarcinoma patients. Br J Cancer. 2015;113(6):878-85.

31. Henson DE, Dittus C, et al. Differential trends in the intestinal and diffuse types of gastric carcinoma in the United States, 1973-2000. Increase in the signet ring cell type. Arch Pathol Lab Med. 2004;128:765-70.

32. Nafteux PR, Lerut TE, et al. Signet ring cells in esophageal and gastroesophageal junction carcinomas have a more aggressive biological behavior. Ann Surg. 2014;260:1023-9.

33. Heger $U$, Blank $S$, et al. Is preoperative chemotherapy followed by surgery the appropriate treatment for signet ring cell containing adenocarcinomas of the esophagogastric junction and stomach? Ann Surg Oncol. 2014;21:1739-48.

34. Patel VR, Hofstetter WL, et al. Signet ring cells in esophageal adenocarcinoma predict poor response to preoperative chemoradiation. Ann Thorac Surg. 2014;98:1064-71.

35. Bekkar S, Gronnier C, et al. The impact of preoperative radiochemotherapy on survival in advanced esophagogastric junction signet ring cell adenocarcinoma. Ann Thorac Surg. 2014;97:303-10.

36. Markar SR, Gronnier C, et al. Role of neoadjuvant treatment in clinical T2N0M0 oesophageal cancer: results from a retrospective multi-center European study. Eur J Cancer. 2016;56:59-68.

37. Speicher PJ, Ganapathi AM, et al. Induction therapy does not improve survival for clinical stage T2N0 esophageal cancer. J Thorac Oncol. 2014;9:1195-201.

38. Samson P, Puri V, et al. Clinical T2N0 esophageal cancer: identifying pretreatment characteristics associated with pathologic upstaging and the potential role for induction therapy. Ann Thorac Surg. 2016;101:2102-11.

39. Raziee HR, Cardoso R, Seevaratnam R, Mahar A, Helyer L, Law $\mathrm{C}$, et al. Systematic review of the predictors of positive margins in gastric cancer surgery and the effect on survival. Gastric Cancer. 2012;15:116-24.

40. Gao F, Chen J, Wang T, Wang G, Zhang Z, Shen L. Incidence of microscopically positive proximal margins in adenocarcinoma of the gastroesophageal junction. PLoS One. 2014;9(2):e88010.

41. Szántó I, Vörös A, Nagy P, Gonda G, Gamal EM, Altorjay Á, et al. Esophageal intramural metastasis from adenocarcinoma of the gastroesophageal junction. Endoscopy. 2002;34(5):418-20.

42. Lam KY, Ma LT, Wong J. Measurement of extent of spread of oesophageal squamous carcinoma by serial sectioning. J Clin Pathol. 1996;49(2):124-9.

43. Khoshnevis J, Moradi A, Azargashb E, Gholizade B. A study of contractility of proximal surgical margin in esophageal cancer. Iran J Cancer Prev. 2011;2011-3. 
44. Mariette C, Castel B, Balon JM, Van Seuningen I, Triboulet JP. Extent of oesophageal resection for adenocarcinoma of the oesophagogastric junction. Eur J Surg Oncol. 2003;29(7):588-93.

45. Ito H, Clancy TE, Osteen RT, Swanson RS, Bueno R, Sugarbaker DJ, Ashley SW, Zinner MJ, Whang EE. Adenocarcinoma of the gastric cardia: what is the optimal surgical approach? J Am Coll Surg. 2004;199(6):880-6.

46. Barbour AP, Rizk NP, Gonen M, Tang L, Bains MS, Rusch VW, et al. Adenocarcinoma of the gastroesophageal junction: influence of esophageal resection margin and operative approach on outcome. Ann Surg. 2007;246(1):1-8.

47. Casson AG, Darnton SJ, Subramanian S, Hiller L. What is the optimal distal resection margin for esophageal carcinoma? Ann Thorac Surg. 2000;69(1):205-9.

48. Chan DS, Reid TD, Howell I, Lewis WG. Systematic review and meta-analysis of the influence of circumferential resection margin involvement on survival in patients with operable oesophageal cancer. Br J Surg. 2013;100(4):456-64. doi:10.1002/bjs.9015 (Epub 2013 Jan 14. Review).

49. Wu J, Chen QX, Teng LS, Krasna MJ. Prognostic significance of positive circumferential resection margin in esophageal cancer: a systematic review and meta-analysis. Ann Thorac Surg. 2014;97(2):446-53. doi:10.1016/j.athoracsur.2013.10.043 (Epub 2013 Dec 21. Review).

50. Parry K, Haverkamp L, Bruijnen RC, Siersema PD, Ruurda JP, van Hillegersberg R. Surgical treatment of adenocarcinomas of the gastro-esophageal junction. Ann Surg Oncol. 2015;22(2):597-603. doi:10.1245/s10434-014-4047-1 (Epub 2014 Sep 5).

51. Pedrazzani C, de Manzoni G, Marrelli D, Giacopuzzi S, Corso G, Minicozzi AM, et al. Lymph node involvement in advanced gastroesophageal junction adenocarcinoma. J Thorac Cardiovasc Surg. 2007;134(2):378-85.

52. Feith M, Stein HJ, Siewert JR. Pattern of lymphatic spread of Barrett's cancer. World J Surg. 2003;27(9):1052-7.

53. Hulsher JBF, van Sandick JW, Offerhaus GJA, Tilanus HW, Obertop H, Van Lanshot JJB. Prospective analysis of the diagnostic yield of extended en bloc resection for adenocarcinoma of the oesophagus or gastric cardia. Br J Surg. 2001;88:715-9.

54. Lerut T, Nafteux P, Moons J, Coosemans W, Decker G, De Leyn $\mathrm{P}$, et al. Three-field lymphadenectomy for carcinoma of the esophagus and gastroesophageal junction in $174 \mathrm{R} 0$ resections: impact on staging, disease-free survival, and outcome: a plea for adaptation of TNM classification in upper-half esophageal carcinoma. Ann Surg. 2004;240(6):962-72 (discussion 972-4).

55. Altorki N, Kent M, Ferrara C, Port J. Three-field lymph node dissection for squamous cell and adenocarcinoma of the esophagus. Ann Surg. 2002;236(2):177-83.

56. Hagen JA, DeMeester SR, Peters JH. Curative resection for esophageal adenocarcinoma analysis of 100 en bloc esophagectomies. Ann Surg. 2001;234(4):520-31.
57. Nigro JJ, Hagen JA, DeMeester TR, et al. Prevalence and location of nodal metastases in distal esophageal adenocarcinoma confined to the wall: implications for therapy. J Thorac Cardiovasc Surg. 1999;117:16-23.

58. Lagarde SM, Cense HA, Hulscher JBF, et al. Prospective analysis of patients with adenocarcinoma of the gastric cardia and lymph node metastasis in the proximal field of the chest. Br J Surg. 2005;92:1404-8

59. Mine S, Sano T, Hiki N, Yamada K, Nunobe S, Yamaguchi T. Lymphadenectomy around the left renal vein in Siewert type II adenocarcinoma of the oesophagogastric junction. Br J Surg. 2013;100(2):261-6.

60. de Manzoni G, Morgagni P, Roviello F, Di Leo A, Saragoni L, Marrelli D, Guglielmi A, Carli A, Folli SCC. Nodal abdominal spread in adenocarcinoma of the cardia. Results of a multicenter prospective study. Gastric Cancer. 1998;1(2):146-51.

61. Aikou T, Shimazu H. Difference in main lymphatic pathways from the lower esophagus and gastric cardia. Jpn J Surg. 1989;19:290-5.

62. Cense HA, Sloof GW, Klaase JM, Bergman JJ, van Hemert FJ, Fockens $\mathrm{P}$, et al. Lymphatic drainage routes of the gastric cardia visualized by lymphoscintigraphy. J Nucl Med. 2004;45:247-52.

63. Hasegawa S, Yoshikawa T, Rino Y, Oshima T, Aoyama T, Hayashi T, et al. Priority of lymph node dissection for Siewert type II/III adenocarcinoma of the esophagogastric junction. Ann Surg Oncol. 2013;20(13):4252-9.

64. Fujitani K, Miyashiro I, Mikata S, et al. Pattern of abdominal nodal spread and optimal abdominal lymphadenectomy for advanced Siewert type II adenocarcinoma of the cardia: results of a multicenter study. Gastric Cancer. 2013;16:301-8.

65. Meier I, Merkel S, Papadopoulos T, Sauer R, Hohenberger WBT. Adenocarcinoma of the esophagogastric junction: the pattern of metastatic lymph node dissemination as a rationale for elective lymphatic target volume definition. Int J Radiat Oncol Biol Phys. 2008;70(5):1408-17.

66. Hosokawa Y, Kinoshita T, Konishi M, Takahashi S, Gotohda N, Kato Y, et al. Clinicopathological features and prognostic factors of adenocarcinoma of the esophagogastric junction according to Siewert classification: experiences at a single institution in Japan. Ann Surg Oncol. 2012;19(2):677-83.

67. Nunobe S, Ohyama S, Sonoo H, Hiki N, Fukunaga T, Seto Y, et al. Benefit of mediastinal and para-aortic lymph-node dissection for advanced gastric cancer with esophageal invasion. J Surg Oncol. 2008;97(5):392-5.

68. Kurokawa Y, Hiki N, Yoshikawa T, Kishi K, Ito Y, Ohi M, et al. Mediastinal lymph node metastasis and recurrence in adenocarcinoma of the esophagogastric junction. Surgery. 2015;157(3):551-5. 